\title{
Androgen Deprivation in Prostate Cancer Patients Treated with Brachytherapy
}

\author{
Georges Hatoum* and Georges Hobeika*
}

Department of Radiation Oncology, University of Miami Miller School of Medicine, $1475 \mathrm{NW} 12^{T H}$ Ave, locator D-31, Miami, Fl. 33136, USA

\begin{abstract}
This article is addressing the role of androgen deprivation therapy (ADT) in prostate brachytherapy. No randomized trials to date evaluating the efficacy of hormonal therapy combined with brachytherapy. Many retrospective trials assessed the role of neoadjuvant androgen deprivation and interstitial permanent prostate brachytherapy in an effort to shrink the prostate gland and to facilitate the brachytherapy procedure in patients with large glands. Hormone ablation has been reported to downsize the prostate gland by $25-40 \%$. Findings in regards to urinary toxicity, mainly urinary retention, related to shrinkage of the prostate are contradictory. Hormonal therapy in combination with brachytherapy is also used for patients with intermediate and high risk features as a result of extrapolation from the external beam radiation therapy data, as brachytherapy alone seems to be suboptimal treatment for men with high-risk prostate cancer. The effect of this combination on biochemical free survival is a matter of debate and varies from one study to another.

Until prospective, randomized studies are done, the role of androgen deprivation therapy (ADT) in conjunction with brachytherapy, specifically in relation to improvement in outcome, remains unclear.
\end{abstract}

Keywords: Prostate, brachytherapy, androgen deprivation.

\section{INTRODUCTION}

The definitive primary treatment for patients with early stage prostate carcinoma includes different options mainly radical prostatectomy, external beam irradiation, and brachytherapy.

The role of androgen deprivation hormone therapy along with external beam radiation therapy has been extensively studied in multiple well-controlled randomized trials with improvement of local failure, disease-free survival and cause-specific survival $[1,2]$.

There are no randomized trials to date evaluating the efficacy of hormonal therapy combined with brachytherapy.

Neoadjuvant hormonal therapy has been used, however, along with prostate brachytherapy in an effort to shrink the prostate gland and avoid pubic arch interference. There are several published studies confirming the value of hormones in this setting [3-6]. Hormone ablation has been reported to downsize the prostate gland by $25-40 \%$ and is used to facilitate the brachytherapy procedure in patients with large glands.

However, many studies demonstrated that many patients who achieved smaller prostate volumes through the use of ADT maintained a significant risk for urinary complications,

*Address correspondence to these authors at the Department of Radiation Oncology, University of Miami Miller School of Medicine, 1475 NW $12^{\mathrm{TH}}$ Ave, locator D-31, Miami, Fl. 33136, USA; Tel: (305)-243-4319; Fax: (305)-243-4363; E-mail: ghatoum1@med.miami.edu and Tel: (305)243-4328; Fax: (305)-243-4363; E-mail: ghobeika@med.miami.edu commensurate with their initial large prostate volume, when compared with a control group of patients who did not receive ADT and were implanted at identical prostate volumes. [7].

Other studies contradict these findings. Crook and colleagues [6] reported that prostate volume and neoadjuvant hormonal therapy were independent predictors of brachytherapy-related urinary retention. A study by Hinerman et al. [8], in conjunction with four additional reports, has demonstrated that hormonal therapy does not significantly increase the risk of post brachytherapy-related urinary retention [9]. However, the duration of hormonal therapy may influence late urinary function. In a study evaluating late urinary function using the Expanded Prostate Cancer Index Composite, statistically significant deterioration was noted in the urinary function and irritation/obstruction domains, with a trend toward increased bother in patients receiving androgen deprivation therapy for more than 6 months (but not less or equal than 6 months) [10]. In patients receiving neoadjuvant hormonal therapy, post treatment IPSS determinations were comparable with those of hormone-naive patients.

The proportion of patients whose IPSS had returned to baseline was nearly identical when stratified by hormonal status. In addition, although hormonally manipulated patients were more likely to require post brachytherapy surgical intervention, the overall need for post brachytherapy TURP/ TUIP was comparable with that of previous reports.

A recent study by Gibbons et al. [11] compared post implant dosimetry in patients with prostate volumes $>50$ 
cc with those with prostate volumes $\leq 50 \mathrm{cc}$. Post implant dosimetry was obtained approximately 4 weeks after brachytherapy.

One-hundred forty-five out of a total of 148 patients had available dosimetry. In the 113 patients with prostate volumes $\leq 50 \mathrm{cc}$ (mean. $35.4 \mathrm{cc}$, range. 14.2-49.7 cc); the mean $D 90$ (dose which covers $90 \%$ of the prostate), $V 100$ (volume of prostate receiving $100 \%$ of the prescribed dose), $V 150$ (volume of prostate receiving $150 \%$ of the prescribed dose), and V200 (volume of prostate receiving $200 \%$ of the prescribed dose) was $128.9 \%, 95.6 \%, 73.9 \%$, and $51.2 \%$, respectively. In the 32 patients with prostate volumes $>50 \mathrm{cc}$ (mean $58.1 \mathrm{cc}$, range $50.2-86.0 \mathrm{cc}$ ); the mean $D 90, V 100$, $V 150$, and $V 200$ was $125.1 \%, 95.2 \%, 68.2 \%$, and $41.7 \%$, respectively. The rectal $V 100$ was $1.0 \mathrm{cc}$ for both cohorts. There was no statistically significant difference between the cohorts with respect to post implant dosimetry for $D 90$, $V 100$, and $V 150$. The $V 200$ for prostate volumes $>50 \mathrm{cc}$ was significantly lower $(p<0.05)$. The study showed that patients with prostate volumes $>50 \mathrm{cc}$ have post implant dosimetry parameters similar to patients with prostate volumes $\leq 50 \mathrm{cc}$ for $D 90, V 100$, and $V 150$; and significantly lower values for $V 200$. These results suggest that patients with large prostate volumes may not need to be routinely placed on hormonal therapy; sparing patients the side effects of hormonal therapy, and sparing the health care system the costs of luteinizing hormone-releasing hormone agonist injections.

Others have used hormonal therapy in combination with brachytherapy for patients with intermediate and high risk features as a result of extrapolation from the external beam radiation therapy data.

A prospective multicenter randomized trial using neoadjuvant hormones for intermediate and high risk brachytherapy patients was attempted in the 1990s, but was closed owing to poor accrual [12].

In a current retrospective study by D'Amico et al. [13], when evaluating prostate cancer specific mortality (PCSM) as the end point, brachytherapy alone seems to be suboptimal treatment for men with high-risk prostate cancer. Moreover, the risk of PCSM was not observed to significantly decrease when either supplemental EBRT or AST were used with brachytherapy, but rather only when the two treatments were combined. This significant reduction in the risk of PCSM was observed despite the fact that men who received both AST and EBRT in addition to brachytherapy had a higher proportion of higher grade and more clinically advanced cancers and therefore a higher baseline risk of PCSM when compared with men who received other therapies. Finally, a decrease in the risk of all-cause mortality (ACM) (AHR= 0.81 ) was noted in the adjusted analysis, although given the competing causes of mortality in this advanced age cohort (median age of 72.7 years), this did not reach significance.

Potters et al. [4] assessed the role of neoadjuvant androgen deprivation and interstitial permanent prostate brachytherapy using a matched-pair analysis of 612 consecutive patients with clinically confined prostate cancer. Patients were treated with either ${ }^{103} \mathrm{Pd}$ or ${ }^{125} \mathrm{I}$ as monotherapy or combined with external radiation. One hundred sixty three (163) patients with prostate glands greater than 60 grams underwent neoadjuvant androgen deprivation to reduce the pros- tate volume. The median duration of hormonal therapy was 3.4 months (range, 1 to 8 months). Two hundred sixty-three (263) patients were matched, with a median follow-up duration of 46 months (range, 24 to 46 months). The five-year PSA relapse free rate for patients treated with combination therapy was 87.1 percent compared with 86.9 percent for those treated with brachytherapy alone. Subgroup analysis by Gleason score groupings, pretreatment PSA, and stage of disease failed to identify any factors for which androgen ablation was beneficial.

In a multivariate analysis, Merrick et al. [14] suggested a slight improvement in the PSA endpoint only for the highrisk subgroup The group evaluated the 5-year biochemical disease-free outcome for men with clinical T1b-T3aNOM0 prostate cancer who underwent permanent prostate brachytherapy using either ${ }^{103} \mathrm{Pd}$ or ${ }^{125} \mathrm{I}$. A total of 77 patients received neoadjuvant androgen deprivation in conjunction with either ${ }^{103} \mathrm{Pd}$ or ${ }^{125} \mathrm{I}$ mono-therapy and 86 patients received neoadjuvant therapy in conjunction with moderatedose external beam radiation therapy and a prostate brachytherapy boost. At a median follow-up of 31 months, patients with low-, intermediate-, and high-risk disease demonstrated 5-year biochemical disease-free rates of 97.1 percent, 97.5 percent, and 84.4 percent, respectively.

In contrast, Stone et al. [15] found in a multivariate analysis that androgen deprivation therapy was the single most important predictor, when compared with dose, risk group, PSA, Gleason score, stage, and isotope. Routine biopsies performed 2 years after brachytherapy showed cancer in $14 \%$ of 181 patients who had no hormones compared with $3.5 \%$ of 115 men treated with neoadjuvant hormones. Fiveyear freedom from biochemical failure was $54 \%$ compared with $79 \%$ for the two groups.

\section{CONCLUSION}

The role of androgen deprivation in prostate brachytherapy is not entirely clear. Other than its proven role as a cytoreductive therapy, the exact mechanism of action whereby hormones enhance the effect of radiation is still a matter of debate. Consequently, it is difficult to predict whether the same survival benefit seen with external beam radiation therapy might be expected with brachytherapy.

Until prospective, randomized studies are done, the role of androgen deprivation therapy (ADT) in conjunction with brachytherapy, specifically in relation to improvement in outcome, remains unclear [16].

\section{REFERENCES}

[1] Bolla M, Gonzalez D, Warde P, et al. Improved survival in patients with locally advanced prostate cancer treated with radiotherapy and goserelin. N Engl J Med 1997; 337: 295-300.

[2] Pilepich MV, Caplan R, Byhardt RW, et al. Phase III trial of androgen suppression using goserelin in unfavorable-prognosis carcinoma of the prostate treated with definitive radiotherapy: report of Radiation Therapy Oncology Group Protocol 85-31. J Clin Oncol 1997; 15(3): 1013-21.

[3] Zelefsky MJ, Leibel ST, Burman CM, et al. Neoadjuvant hormonal therapy improves the therapeutic ratio in patients with bulky prostatic cancer treated with three-dimensional conformal radiation therapy. Int J Radiat Oncol Biol Phys 1994; 29: 4: 755-61.

[4] Potters L, Torre T, Ashley R, et al. Examining the role of neoadjuvant androgen deprivation in patients undergoing prostate brachytherapy. J Clin Oncol 2000; 6: 1187-92. 
[5] Blank KR, Whittington R, Arjomandy B, et al. Neoadjuvant androgen deprivation prior to transperineal prostate brachytherapy: Smaller volumes, less morbidity. Cancer J Sci Am 1999; 6: 370-3.

[6] Crook J, McLean M, Catton C, et al. Factors influencing risk of acute urinary retention after TRUS-guided permanent prostate seed implantation. Int J Radiat Oncol Biol Phys 2002; 52: 453-60.

[7] Petit J, Gluck C, Kiger III WS, et al. Androgen-deprivationmediated cytoreduction before interstitial brachytherapy for prostate cancer does not abrogate the elevated risk of urinary morbidity associated with larger interstitial prostate volume. Brachytherapy 2007; 4: 267-71.

[8] Hinerman A, Merrick G, Butler W, et al. Androgen deprivationinduced changes in prostate anatomy predict urinary morbidity after permanent interstitial brachytherapy. Int J Radiat Oncol Biol Phys 2004; 59(5): 1367-82.

[9] Terk MD, Stock RG, Stone NN. Identification of patients at increased risk for prolonged urinary retention following radioactive seed implantation of the prostate. J Urol 1998; 160: 1379-82.

[10] Locke J, Ellis W, Wallner K, et al. Risk factors for acute urinary retention requiring temporary intermittent catheterization after prostate brachytherapy: A prospective study. Int J Radiat Oncol Biol Phys 2002; 52: 712-9.
[11] Gibbons E, Jacobs B, Smith R., et al. Dosimetric outcomes in prostate brachytherapy: Is downsizing the prostate with androgen deprivation necessary? Brachytherapy 2009; 8(3): 304-8.

[12] Sylvester J, Blasko J, Grimm P, et al. Impact of short-course androgen ablation on the biochemical progression-free survival of high-risk prostate cancer patients managed with permanent brachytherapy. J Brachyther Int 2001; 17: 173-80.

[13] D'Amico AV, Moran BJ, Braccioforte MH, et al. Risk of death from prostate cancer after brachytherapy alone or with radiation, androgen suppression therapy, or both in men with high-risk disease. J Clin Oncol 2009; 27(24): 3923-8.

[14] Merrick GS, Butler WM, Gal breath RW, et al. Does hormonal manipulation in conjunction with permanent interstitial brachytherapy, with or without supplemental external beam irradiation, improve the biochemical outcome for men with intermediate or high-risk prostate cancer? BJU Int 2003; 91: 23a.

[15] Stone NN, Stock RG, Unger P. Effects of neoadjuvant hormonal therapy on prostate biopsy results after $125 \mathrm{I}$ and $103 \mathrm{Pd}$ seed implantation. Mol Urol 2000; 3: 163-70.

[16] Grimm PD, Blasko JC, Sylvester JE, et al. 10-year biochemical (prostate-specific antigen) control of prostate cancer with (125) I brachytherapy. Int J Radiat Oncol Biol Phys 2001; 51: 31-40.

Received: October 12, 2009

Revised: December 19, 2009

Accepted: December 19, 2009

(c) Hatoum and Hobeika; Licensee Bentham Open.

This is an open access article licensed under the terms of the Creative Commons Attribution Non-Commercial License (http://creativecommons.org/licenses/ by-nc/3.0/) which permits unrestricted, non-commercial use, distribution and reproduction in any medium, provided the work is properly cited. 